\title{
On set-valued contractions of Nadler type in tus- G-cone metric spaces
}

\author{
Chi-Ming Chen
}

Correspondence: ming@mail.nhcue. edu.tw

Department of Applied

Mathematics, National Hsinchu University of Education, No. 521

Nanda Rd., Hsinchu City 300,

Taiwan

\begin{abstract}
In this article, for a tus-G-cone metric space $(X, G)$ and for the family $\mathcal{A}$ of subsets of $X$, we introduce a new notion of the $t v s-\mathcal{H}$ - cone metric $\mathcal{H}$ with respect to $G$, and we get a fixed result for the stronger Meir-Keeler-G-cone-type function in a complete tus-G-cone metric space $(\mathcal{A}, \mathcal{H})$ Our result generalizes some recent results due to Dariusz Wardowski and Radonevic' et al.
\end{abstract}

MSC: 47H10; 54C60; 54H25; 55M20.

Keywords: fixed point theorem, stronger Meir-Keeler-G-cone-type function, tus-Gcone metric space

\section{Introduction and preliminaries}

Recently, Huang and Zhang [1] introduced the concept of cone metric space by replacing the set of real numbers by an ordered Banach space, and they showed some fixed point theorems of contractive type mappings on cone metric spaces. The category of cone metric spaces is larger than metric spaces. Subsequently many authors like Abbas and Jungck [2] had generalized the results of Huang and Zhang [1] and studied the existence of common fixed points of a pair of self mappings satisfying a contractive type condition in the framework of normal cone metric spaces. However, authors like Jankovic' et al. [3], Rezapour and Hamlbarani [4] studied the existence of common fixed points of a pair of self and nonself mappings satisfying a contractive type condition in the situation in which the cone does not need to be normal. Many authors studied this subject and many results on fixed point theory are proved (see e.g., [4-15]).

Recently, Du [16] introduced the concept of tvs-cone metric and tvs-cone metric space to improve and extend the concept of cone metric space in the sense of Huang and Zhang [1]. Later, in the articles [16-19], the authors tried to generalize this approach by using cones in topological vector spaces tvs instead of Banach spaces. However, it should be noted that an old result shows that if the underlying cone of an ordered tus is solid and normal, then such tus must be an ordered normed space. Thus, proper generalizations when passing from norm-valued cone metric spaces to tvs-valued cone metric spaces can be obtained only in the case of nonnormal cones (for details, see [19]).

We recall some definitions and results of the tus-cone metric spaces that introduced in $[19,20]$, which will be needed in the sequel.

(c) 2012 Chen; licensee Springer. This is an Open Access article distributed under the terms of the Creative Commons Attribution License (http://creativecommons.org/licenses/by/2.0), which permits unrestricted use, distribution, and reproduction in any medium, provided the original work is properly cited. 
Let $E$ be a real Hausdorff topological vector space (tvs for short) with the zero vector $\theta$. A nonempty subset $P$ of $E$ is called a convex cone if $P+P \subseteq P$ and $\lambda P \subseteq P$ for $\lambda \geq$ 0 . A convex cone $P$ is said to be pointed (or proper) if $P \cap(-P)=\{\theta\} ; P$ is normal (or saturated) if $E$ has a base of neighborhoods of zero consisting of order-convex subsets. For a given cone $P \subseteq E$, we can define a partial ordering $\leqslant$ with respect to $P$ by $x \leqslant y$ if and only if $y-x \in P ; x<y$ will stand for $x \leqslant y$ and $x \neq y$, while $x \ll y$ will stand for $y-x \in \operatorname{int} P$, where int $P$ denotes the interior of $P$. The cone $P$ is said to be solid if it has a nonempty interior.

In the sequel, $E$ will be a locally convex Hausdorff $t v s$ with its zero vector $\theta, P$ a proper, closed, and convex pointed cone in $E$ with int $P \neq \varphi$ and $\preccurlyeq$ a partial ordering with respect to $P$.

Definition $1[16,18,19]$ Let $X$ be a nonempty set and $(E, P)$ an ordered tvs. A vectorvalued function $d: X \times X \rightarrow E$ is said to be a tvs-cone metric, if the following conditions hold:

$\left(C_{1}\right) \forall_{x, y \in X, x \neq y} \theta \leqslant d(x, y)$;

$\left(C_{2}\right) \forall_{x, y \in X} d(x, y)=\theta \Leftrightarrow x=y$;

(C) $\forall_{x, y \in X} d(x, y)=d(y, x)$;

$\left(C_{4}\right) \forall_{x, y, z \in X} d(x, z) \leqslant d(x, y)+d(y, z)$.

Then the pair $(X, d)$ is called a tvs-cone metric space.

Definition $2[16,18,19]$ Let $(X, d)$ be a tvs-cone metric space, $x \in X$ and $\left\{x_{n}\right\}$ a sequence in $X$.

(1) $\left\{x_{n}\right\}$ tus-cone converges to $x$ whenever for every $c \in E$ with $\theta \ll c$, there exists $n_{0} \in \mathbb{N}$ such that $d\left(x_{n}, x\right) \ll c$ for all $n \geq n_{0}$. We denote this by cone- $\lim _{n \rightarrow \infty} x_{n}=x$;

(2) $\left\{x_{n}\right\}$ is a tus-cone Cauchy sequence whenever for every $c \in E$ with $\theta \ll c$, there exists $n_{0} \in \mathbb{N}$ such that $d\left(x_{n}, x_{m}\right) \ll c$ for all $n, m \geq n_{0}$;

(3) $(X, d)$ is tus-cone complete if every tvs-cone Cauchy sequence in $X$ is tvs-cone convergent in $X$.

Remark 1 Clearly, a cone metric space in the sense of Huang and Zhang [1] is a special case of tus-cone metric spaces when $(X, d)$ is a tus-cone metric space with respect to a normal cone $P$.

Remark 2 [19-21] Let $(X, d)$ be a tus-cone metric space with a solid cone P. The following properties are often used, particularly in the case when the underlying cone is nonnormal.

(p1) If $u \leqslant v$ and $v \ll w$, then $u \ll w$;

(p2) If $u \ll v$ and $v \leqslant w$, then $u \ll w$;

(p3) If $u \ll v$ and $v \ll w$, then $u \ll w$;

(p4) If $\theta \leqslant u \ll c$ for each $c \in$ int $P$, then $u=\theta$;

(p5) If $a \leqslant b+c$ for each $c \in$ intP, then $a \leqslant b$;

(p6) If $E$ is tus with a cone $P$, and if $a \leqslant \lambda a$ where $a \in P$ and $\lambda \in[0,1)$, then $a=\theta$;

(p7) If $c \in \operatorname{int} P, a_{n} \in E$ and $a_{n} \rightarrow \theta$ in locally convex tvs $E$, then there exists $n_{0} \in \mathbb{N}$ such that $a_{n} \ll c$ for all $n>n_{0}$.

Metric spaces are playing an important role in mathematics and the applied sciences. To overcome fundamental laws in Dhage's theory of generalized metric spaces [22], flaws that invalidate most of the results claimed for these spaces, Mustafa and Sims [23] introduced a more appropriate and robust notion of a generalized metric space as follows: 
Definition 3 [23] Let $X$ be a nonempty set, and let $G: X \times X \times X \rightarrow[0, \infty)$ be a function satisfying the following axioms:

(G1) $\forall_{x, y, z \in X} G(x, y, z)=0 \Leftrightarrow x=y=z$;

(G2) $\forall_{x, y \in X, x \neq y} G(x, x, y)>0$;

(G3) $\forall_{x, y, z \in X} G(x, y, z) \geq G(x, x, y)$;

(G4) $\forall_{x, y, z \in X} G(x, y, z)=G(x, z, y)=G(z, y, x)=\ldots$ (symmetric in all three variables);

(G5) $\forall_{x, y, z, w \in X} G(x, y, z) \leq G(x, w, w)+G(w, y, z)$.

Then the function $G$ is called a generalized metric, or, more specifically a G-metric on $X$, and the pair $(X, G)$ is called a G-metric space.

By using the notions of generalized metrics and tvs-cone metrics, we introduced the below notion of tvs-generalized-cone metrics.

Definition 4 Let $X$ be a nonempty set and $(E, P)$ an ordered tus, and let $G: X \times X \times$ $X \rightarrow E$ be a function satisfying the following axioms:

(G1) $\forall_{x, y, z \in X} G(x, y, z)=\theta$ if and only if $x=y=z$;

(G2) $\forall_{x, y \in X, x \neq y} \theta \ll G(x, x, y)$;

(G3) $\forall_{x, y, z \in X} G(x, x, y) \leqslant G(x, y, z)$;

(G4) $\forall_{x, y, z \in X} G(x, y, z)=G(x, z, y)=G(z, y, x)=\ldots$ (symmetric in all three variables);

(G5) $\forall_{x, y, z, w \in X} G(x, y, z) \leqslant G(x, w, w)+G(w, y, z)$.

Then the function $G$ is called a tvs-generalized-cone metric, or, more specifically a tvs-G-cone metric on $X$, and the pair $(X, G)$ is called a tus-G-cone metric space.

Definition 5 Let $(X, G)$ be a tvs-G-cone metric space, $x \in X$ and $\left\{x_{n}\right\}$ a sequence in $X$.

(1) $\left\{x_{n}\right\}$ tvs-G-cone converges to $x$ whenever for every $c \in E$ with $\theta \ll c$, there exists $n_{0} \in \mathbb{N}$ such that $G\left(x_{n}, x_{m}, x\right) \ll c$ for all $m, n \geq n_{0}$. Here $x$ is called the limit of the sequence $\left\{x_{n}\right\}$ and is denoted by G-cone-lim ${ }_{n \rightarrow \infty} x_{n}=x$;

(2) $\left\{x_{n}\right\}$ is a tus-G-cone Cauchy sequence whenever for every $c \in E$ with $\theta \ll c$, there exists $n_{0} \in \mathbb{N}$ such that $G\left(x_{n}, x_{m}, x_{l}\right) \ll c$ for all $n, m, l \geq n_{0}$;

(3) $(X, G)$ is tvs-G-cone complete if every tvs-G-cone Cauchy sequence in $X$ is tvs-Gcone convergent in $X$.

Proposition 1 Let $(X, G)$ be a tvs-G-cone metric space, $x \in X$ and $\left\{x_{n}\right\}$ a sequence in $X$. The following are equivalent

(i) $\left\{x_{n}\right\}$ tvs-G-cone converges to $x$;

(ii) $G\left(x_{n}, x_{n}, x\right) \rightarrow \theta$ as $n \rightarrow \infty$;

(iii) $G\left(x_{n}, x, x\right) \rightarrow \theta$ as $n \rightarrow \infty$;

$($ iv $) G\left(x_{n}, x_{m}, x\right) \rightarrow \theta$ as $n, m \rightarrow \infty$.

We also recall the notion of Meir-Keeler type function (see [24]). A function $\phi:[0$, $\infty) \rightarrow[0, \infty)$ is said to be a Meir-Keeler type function, if $\phi$ satisfies the following condition:

$$
\forall \eta>0 \exists \delta>0 \forall t \in[0, \infty)(\eta \leq t<\delta+\eta \Rightarrow \varphi(t)<\eta) .
$$

We now define a new notion of stronger Meir-Keeler type function, as follows:

Definition 6 We call $\phi:[0, \infty) \rightarrow[0,1)$ a stronger Meir-Keeler type function if the function $\phi$ satisfies the following condition:

$$
\forall \eta>0 \exists \delta>0 \exists \gamma_{\eta} \in[0,1) \forall t \in[0, \infty)\left(\eta \leq t<\delta+\eta \Rightarrow \varphi(t)<\gamma_{\eta}\right) .
$$


And, we introduce the below concept of the stronger Meir-Keeler tvs-G-cone-type function in a tvs-G-cone metric space.

Definition 7 Let $(X, G)$ be a tus-G-cone metric space with a solid cone P. We call $\phi$ : $P \rightarrow[0,1)$ a stronger Meir-Keeler tvs-G-cone-type function in $X$ if the function $\phi$ satisfies the following condition:

$$
\forall \eta \gg \theta \exists \delta \gg \theta \exists \gamma_{\eta} \in[0,1) \quad \forall x, y, z \in X\left(\eta \preccurlyeq G(x, y, z) \ll \delta+\eta \Rightarrow \varphi(G(x, y, z))<\gamma_{\eta}\right) .
$$

The Nadler's results [25] concerning set-valued contractive mappings in metric spaces became the inspiration for many authors in the metric fixed point theory (see for example [26-28]). Particularly Wardowski [29] established a new cone metric $\mathcal{H}: \mathcal{A} \times \mathcal{A} \rightarrow E$ for a cone metric space $(X, d)$ and for the family $\mathcal{A}$ of subsets of $X$, and introduced the concept of set-valued contraction of Nadler type and prove a fixed point theorem. Later, in [21], the concept of set-valued contraction of Nadler type in the setting of $t v s$-cone spaces was introduced and a fixed point theorem in the setting of tus-cone spaces with respect to a solid cone was proved.

In this article, for a $t v s-G$-cone metric space $(X, G)$ and for the family $\mathcal{A}$ of subsets of $X$, we introduce a new notion of the $t v s-\mathcal{H}$ - cone metric $\mathcal{H}$ with respect to $G$, and we get a fixed result for the stronger Meir-Keeler type function in a complete tvs-generalized-cone metric space $(\mathcal{A}, \mathcal{H})$. Our result generalizes some recent results due to Radonevic' et al. [21] and Dariusz Wardowski [29].

\section{Main results}

Let $E$ be a locally convex Hausdorff $t v s$ with its zero vector $\theta, P$ a proper, closed, and convex pointed cone in $E$ with $\operatorname{int} P \neq \varphi$ and $\leqslant$ a partial ordering with respect to $P$. We introduce the below notion of the $t v s-\mathcal{H}$ - cone metric $\mathcal{H}$ with respect to $t v s-G$-cone metric G.

Definition 8 Let $(X, G)$ be a tvs-G-cone metric space with a solid cone $P$ and let $\mathcal{A}$ be a collection of nonempty subsets of $X . A$ map $\mathcal{H}: \mathcal{A} \times \mathcal{A} \times \mathcal{A} \rightarrow E$ is called a tvs $-\mathcal{H}$ - cone metric with respect to $\mathrm{G}$ if for any $A_{1}, A_{2}, A_{3} \in \mathcal{A}$ the following conditions hold:

$$
\begin{aligned}
& \left(H_{1}\right) \mathcal{H}\left(A_{1}, A_{2}, A_{3}\right)=\theta \Rightarrow A_{1}=A_{2}=A_{3} ; \\
& \left(H_{2}\right) \mathcal{H}\left(A_{1}, A_{2}, A_{3}\right)=\mathcal{H}\left(A_{1}, A_{2}, A_{3}\right)=\mathcal{H}\left(A_{1}, A_{2}, A_{3}\right)=\cdots \text { (symmetry in all variables); } \\
& \text { (H } \left.H_{3}\right) \mathcal{H}\left(A_{1}, A_{2}, A_{3}\right) \preccurlyeq \mathcal{H}\left(A_{1}, A_{2}, A_{3}\right) ; \\
& \text { (H } \left.H_{4}\right) \forall_{\varepsilon \in E, \theta \ll \varepsilon} \forall_{x \in A_{1}, y \in A_{2}} \exists_{z_{\epsilon} A_{3}} G(x, y, z) \preccurlyeq \mathcal{H}\left(A_{1}, A_{2}, A_{3}\right)+\varepsilon ; \\
& \text { (H } \left.H_{5}\right) \text { one of the following is satisfied: } \\
& \text { (i) } \forall_{\varepsilon \in E, \theta \ll \varepsilon} \exists_{x_{\in} A_{1}} \forall_{y \in A_{2}, z \in A_{3}} \mathcal{H}\left(A_{1}, A_{2}, A_{3}\right) \preccurlyeq G(x, y, z)+\varepsilon ; \\
& \text { (ii) } \forall_{\varepsilon \in E, \theta \ll \varepsilon} \exists_{x_{\epsilon} A_{2}} \forall_{x \in A_{1}, z \in A_{3}} \mathcal{H}\left(A_{1}, A_{2}, A_{3}\right) \preccurlyeq G(x, y, z)+\varepsilon ; \\
& \text { (iii) } \forall_{\varepsilon \in E, \theta \ll \varepsilon} \exists_{z_{\epsilon} A_{3}} \forall_{y \in A_{2}, z \in A_{1}} \mathcal{H}\left(A_{1}, A_{2}, A_{3}\right) \preccurlyeq G(x, y, z)+\varepsilon .
\end{aligned}
$$

Lemma 1 Let $(X, G)$ be a tvs-G-cone metric space with a solid cone $P$ and let $\mathcal{A}$ be a collection of nonempty subsets of $X . \mathcal{A} \neq \phi$. If $\mathcal{H}: \mathcal{A} \times \mathcal{A} \times \mathcal{A} \rightarrow$ Eis a tvs $-\mathcal{H}-$ cone metric with respect to $G$, then pair $(\mathcal{A}, \mathcal{H})$ is a tus-G-cone metric space.

Proof Let $\left\{\varepsilon_{n}\right\} \subset E$ be a sequence such that $\theta \ll \varepsilon_{n}$ for all $n \in \mathbb{N}$ and G-cone-lim $\lim _{n \rightarrow \infty}$ $\varepsilon_{n}=\theta$. Take any $A_{1}, A_{2}, A_{3} \in \mathcal{A}$ and $x \in A_{1}, y \in A_{2}$. From $\left(H_{4}\right)$, for each $n \in \mathbb{N}$, there exists $z_{n} \in A_{3}$ such that 


$$
G\left(x, y, z_{n}\right) \preccurlyeq \mathcal{H}\left(A_{1}, A_{2}, A_{3}\right)+\varepsilon_{n} .
$$

Therefore, $\mathcal{H}\left(A_{1}, A_{2}, A_{3}\right)+\varepsilon_{n} \in P$ for each $n \in \mathbb{N}$. By the closedness of $P$, we conclude that $\theta \leqslant \mathcal{H}\left(A_{1}, A_{2}, A_{3}\right)$.

Assume that $A_{1}=A_{2}=A_{3}$. From $H_{5}$, we obtain $\mathcal{H}\left(A_{1}, A_{2}, A_{3}\right) \preccurlyeq \varepsilon_{n}$ for any $n \in \mathbb{N}$. So $\mathcal{H}\left(A_{1}, A_{2}, A_{3}\right)=\theta$.

Let $A_{1}, A_{2}, A_{3}, A_{4} \in \mathcal{A}$. Assume that $A_{1}, A_{2}, A_{3}$ satisfy the condition $\left(H_{5}\right)(i)$. Then for each $n \in \mathbb{N}$, there exists $x_{n} \in A_{1}$ such that $\mathcal{H}\left(A_{1}, A_{2}, A_{3}\right) \preccurlyeq G\left(x_{n}, y, z\right)+\varepsilon_{n}$ for all $y \in$ $A_{2}$ and $z \in A_{3}$. From $\left(H_{4}\right)$, there exists a sequence $\left\{w_{n}\right\} \subset A_{4}$ satisfying $G\left(x_{n}, w_{n}, w_{n}\right) \preccurlyeq \mathcal{H}\left(A_{1}, A_{4}, A_{4}\right)+\varepsilon_{n}$ for every $n \in \mathbb{N}$. Obviously for any $y \in A_{2}$ and any $z$ $\in A_{3}$ and $n \in \mathbb{N}$, we have

$$
\begin{aligned}
\mathcal{H}\left(A_{1}, A_{2}, A_{3}\right) & \preccurlyeq G\left(x_{n}, y, z\right)+\varepsilon_{n} \\
& \preccurlyeq G\left(x_{n}, w_{n}, w_{n}\right)+G\left(w_{n}, y, z\right)+\varepsilon_{n} .
\end{aligned}
$$

Now for each $n \in \mathbb{N}$, there exists $y_{n} \in A_{2}, z_{n} \in A_{3}$ such that $G\left(w_{n}, y_{n}, z_{n}\right) \preccurlyeq \mathcal{H}\left(A_{4}, A_{2}, A_{3}\right)+\varepsilon_{n}$. Consequently, we obtain that for each $n \in \mathbb{N}$

$$
\mathcal{H}\left(A_{1}, A_{2}, A_{3}\right) \preccurlyeq \mathcal{H}\left(A_{1}, A_{4}, A_{4}\right)+\mathcal{H}\left(A_{4}, A_{2}, A_{3}\right)+3 \varepsilon_{n} .
$$

Therefore,

$$
\mathcal{H}\left(A_{1}, A_{2}, A_{3}\right) \preccurlyeq \mathcal{H}\left(A_{1}, A_{4}, A_{4}\right)+\mathcal{H}\left(A_{4}, A_{2}, A_{3}\right) .
$$

In the case when $\left(H_{5}\right)(i i)$ or $\left(H_{5}\right)(i i i)$ hold, we use the analog method. $\square$

Our main result is the following.

Theorem 1 Let $(X, G)$ be a tus-G-cone complete metric space with a solid cone $P$ and let $\mathcal{A} b e$ a collection of nonempty closed subsets of $X, \mathcal{A} \neq \phi$, and let $\mathcal{H}: \mathcal{A} \times \mathcal{A} \times \mathcal{A} \rightarrow$ Ebe a tvs $-\mathcal{H}$-conemetric with respect to $G$. If the mapping $T: X \rightarrow$ Asatisfies the condition that exists a stronger Meir-Keeler tvs-G-cone-type function $\phi: P \rightarrow[0,1)$ such that for all $x, y, z \in X$ holds

$$
\mathcal{H}(T x, T y, T z) \preccurlyeq \varphi(G(x, y, z)) \cdot G(x, y, z),
$$

then $T$ has a fixed point in $X$.

Proof. Let us choose $x_{0} \in X$ arbitrarily and $x_{1} \in T x_{0}$. If $G\left(x_{0}, x_{0}, x_{1}\right)=\theta$, then $x_{0}=x_{1}$ $\in T\left(x_{0}\right)$, and we are done. Assume that $G\left(x_{0}, x_{0}, x_{1}\right) \ll \theta$. Put $G\left(x_{0}, x_{0}, x_{1}\right)=\eta_{0}, \eta_{0} \gg$ $\theta$. By the definition of the stronger Meir-Keeler tvs-G-cone-type function $\phi: P \rightarrow[0$, $1)$, corresponding to $\eta_{0}$ use, there exist $\delta_{0} \gg \theta$ and $\gamma_{\eta_{0}} \in(0,1)$ with $\eta_{0} \leqslant G\left(x_{0}, x_{0}, x 1\right)$ $\prec \eta_{0}+\delta_{0}$ such that $\varphi\left(G\left(x_{0}, x_{0}, x_{1}\right)\right)<\gamma_{\eta_{0}}$. Let $\varepsilon \in \operatorname{intP}$ and $\varepsilon_{1} \in E$ such that $\theta \ll \varepsilon_{1}$ and $\varepsilon_{1} \preccurlyeq \gamma_{\eta_{0}} \cdot \varepsilon$. Taking into account (1) and $\left(H_{4}\right)$, there exists $x_{2} \in T x_{1}$ such that

$$
\begin{aligned}
G\left(x_{1}, x_{1}, x_{2}\right) & \preccurlyeq \mathcal{H}\left(T x_{0}, T x_{0}, T x_{1}\right)+\varepsilon_{1} \\
& \preccurlyeq \varphi\left(G\left(x_{0}, x_{0}, x_{1}\right)\right) \cdot G\left(x_{0}, x_{0}, x_{1}\right)+\varepsilon_{1} \\
& \preccurlyeq \gamma_{\eta_{0}} \cdot G\left(x_{0}, x_{0}, x_{1}\right)+\varepsilon_{1} .
\end{aligned}
$$

Now, put $G\left(x_{1}, x_{1}, x_{2}\right)=\eta_{1}, \eta_{1} \gg \theta$. By the definition of the stronger Meir-Keeler tvs-G-cone-type function $\phi: P-[0,1)$, corresponding to $\eta_{1}$ use, there exist $\delta_{1} \gg \theta$ and $\gamma_{\eta_{1}} \in(0,1)$ with $\eta_{1} \leqslant G\left(x_{1}, x_{1}, x_{2}\right) \prec \eta_{1}+\delta_{1}$ such that $\varphi\left(G\left(x_{1}, x_{1}, x_{2}\right)\right)<\gamma_{\eta_{1}}$. Put $\alpha_{0}=\gamma_{\eta_{0}}$ and $\alpha_{1}=\max \left\{\gamma_{\eta_{0}}, \gamma_{\eta_{1}}\right\}$. Then $\alpha_{0}, \alpha_{1} \in(0,1)$ and

$$
\varphi\left(G\left(x_{0}, x_{0}, x_{1}\right)\right)<\gamma_{\eta_{0}} \leq \alpha<1 \text { and } \varphi\left(G\left(x_{1}, x_{1}, x_{2}\right)\right)<\gamma_{\eta_{1}} \leq \alpha_{1}<1 .
$$


Let $\varepsilon_{2} \in E$ such that $\theta \ll \varepsilon_{2}$ and $\varepsilon_{2} \preccurlyeq \gamma_{\eta_{1}}^{2} \cdot \varepsilon$. Then

$$
\varepsilon_{1} \preccurlyeq \alpha_{1} \cdot \varepsilon \text { and } \varepsilon_{2} \preccurlyeq \alpha_{1}^{2} \cdot \varepsilon \text {. }
$$

Taking into account (1), (2), and $\left(H_{4}\right)$, there exists $x_{3} \in T x_{2}$ such that

$$
\begin{aligned}
G\left(x_{2}, x_{2}, x_{3}\right) & \preccurlyeq \mathcal{H}\left(T x_{1}, T x_{1}, T x_{2}\right)+\varepsilon_{2} \\
& \preccurlyeq \varphi\left(G\left(x_{1}, x_{1}, x_{2}\right)\right) \cdot G\left(x_{1}, x_{1}, x_{2}\right)+\varepsilon_{2} \\
& \preccurlyeq \alpha_{1} \cdot G\left(x_{1}, x_{1}, x_{2}\right)+\varepsilon_{2} \\
& \preccurlyeq \alpha_{1}\left(\alpha_{1} \cdot G\left(x_{0}, x_{0}, x_{1}\right)+\varepsilon_{1}\right)+\varepsilon_{2} \\
& \preccurlyeq \alpha_{1}^{2} \cdot G\left(x_{0}, x_{0}, x_{1}\right)+\alpha_{1} \cdot \varepsilon_{1}+\varepsilon_{2} \\
& \preccurlyeq \alpha_{1}^{2} \cdot G\left(x_{0}, x_{0}, x_{1}\right)+2 \alpha_{1}^{2} \cdot \varepsilon .
\end{aligned}
$$

We continue in this manner. In general, for $x_{n}, n \in \mathbb{N}, x_{n+1}$ is chosen such that $x_{n+1}$ $\in T x_{n}$. Put $G\left(x_{n}, x_{n}, x_{n+1}\right)=\eta_{n}, \eta_{n} \gg \theta$. By the definition of the stronger Meir-Keeler tvs-G-cone-type function $\phi: P \rightarrow[0,1)$, corresponding to $\eta_{n}$ use, there exist $\delta_{n} \gg \theta$ and $\gamma_{\eta_{n}} \in(0,1)$ with $\eta_{n} \leqslant G\left(x_{n}, x_{n}, x_{n+1}\right) \prec \eta_{n}+\delta_{n}$ such that $\varphi\left(G\left(x_{n}, x_{n}, x_{n+1}\right)\right)<\gamma_{\eta_{n}}$. Put $\alpha_{n}=\max \left\{\gamma_{\eta_{0}}, \gamma_{\eta_{1}}, \ldots, \gamma_{\eta_{n}}\right\}, n \in \mathbb{N}$. Then $\alpha_{n} \in(0,1)$ and

$$
\varphi\left(G\left(x_{i}, x_{i}, x_{i+1}\right)\right)<\gamma_{\eta_{i}} \leq \alpha_{n}<1, \quad \text { for all } i \in\{0,1,2, \ldots, n\} .
$$

On the other hand, for each $n \in \mathbb{N}$, corresponding to $\gamma_{\eta_{n}}$ use, we choose $\varepsilon_{n+1} \in E$ such that $\theta \ll \varepsilon_{n+1}$ and $\varepsilon_{n+1} \preccurlyeq \gamma_{\eta_{n}}^{n+1} \cdot \varepsilon$. Then

$$
\varepsilon_{n+1} \preccurlyeq \alpha_{n}^{n+1} \cdot \varepsilon .
$$

From above argument, we can construct a sequence $\left\{x_{n}\right\}$ in $X$, a non-decreasing sequence $\left\{\alpha_{n}\right\}$ and a sequence $\left\{\varepsilon_{n}\right\}$ recursively as follow:

$$
\begin{gathered}
x_{n+1} \in T x_{n}, \\
\alpha_{n}=\max \left\{\gamma_{\eta_{0}}, \gamma_{\eta_{1}}, \ldots, \gamma_{\eta_{n}}\right\}<1, \\
\varepsilon_{n+1} \preccurlyeq \gamma_{\eta_{n}}^{n+1} \cdot \varepsilon \preccurlyeq \alpha_{n}^{n+1} \cdot \varepsilon,
\end{gathered}
$$

for all $n \in \mathbb{N} \cup\{0\}$.

And, we have that for each $n \in \mathbb{N} \cup\{0\}$

$$
G\left(x_{n+1}, x_{n+1}, x_{n+2}\right) \preccurlyeq \mathcal{H}\left(T x_{n}, T x_{n}, T x_{n+1}\right)+\varepsilon_{n+1} .
$$

Taking into account (4), (5), and $\left(H_{4}\right)$, there exists $x_{n+2} \in T x_{n+1}$ such that

$$
\begin{aligned}
G\left(x_{n+1}, x_{n+1}, x_{n+2}\right) & \preccurlyeq \mathcal{H}\left(T x_{n}, T x_{n}, T x_{n+1}\right)+\varepsilon_{n+1} \\
& \preccurlyeq \varphi\left(G\left(x_{n}, x_{n}, x_{n+1}\right)\right) \cdot G\left(x_{n}, x_{n}, x_{n+1}\right)+\varepsilon_{n+1} \\
& \preccurlyeq \alpha_{n} \cdot G\left(x_{n}, x_{n}, x_{n+1}\right)+\alpha_{n}^{n+1} \cdot \varepsilon \\
& \preccurlyeq \alpha_{n}\left[\mathcal{H}\left(T x_{n-1}, T x_{n-1}, T x_{n}\right)+\varepsilon_{n}\right]+\alpha_{n}^{n+1} \cdot \varepsilon \\
& \preccurlyeq \alpha_{n}\left[\varphi\left(G\left(x_{n-1}, x_{n-1}, x_{n}\right)\right) \cdot G\left(x_{n}, x_{n}, x_{n+1}\right)+\varepsilon_{n}\right]+\alpha_{n}^{n+1} \cdot \varepsilon \\
& \preccurlyeq \alpha_{n}\left[\alpha_{n} \cdot G\left(x_{n}, x_{n}, x_{n+1}\right)+\varepsilon_{n}\right]+\alpha_{n}^{n+1} \cdot \varepsilon \\
& \preccurlyeq \alpha_{n}^{2} \cdot G\left(x_{n}, x_{n}, x_{n+1}\right)+\alpha_{n} \cdot \varepsilon_{n}+\alpha_{n}^{n+1} \cdot \varepsilon \\
& \preccurlyeq \alpha_{n}^{2} \cdot G\left(x_{n}, x_{n}, x_{n+1}\right)+2 \alpha_{n}^{n+1} \cdot \varepsilon \\
& \preccurlyeq \cdots \cdots \\
& \preccurlyeq \alpha_{n}^{n+1} \cdot G\left(x_{0}, x_{0}, x_{1}\right)+(n+1) \alpha_{n}^{n+1} \cdot \varepsilon .
\end{aligned}
$$


Let $m, n \in \mathbb{N}$ be such that $m>n$. From (6) we conclude that

$$
\begin{aligned}
G\left(x_{n}, x_{n}, x_{m}\right) & \preccurlyeq \sum_{j=n}^{m-1} G\left(x_{j}, x_{j}, x_{j+1}\right) \\
& \preccurlyeq \sum_{j=n}^{m-1}\left[\alpha_{j-1}^{j} \cdot G\left(x_{0}, x_{0}, x_{1}\right)+j \alpha_{j-1}^{j} \cdot \varepsilon\right] .
\end{aligned}
$$

From above argument and the inequality (7), we put $\alpha=\max \left\{\alpha_{n-1}, \alpha_{n}, \alpha_{n+1}, \ldots, \alpha_{m-}\right.$ 2). Then, we get $\alpha=\alpha_{m-2}<1$ and

$$
\begin{aligned}
G\left(x_{n}, x_{n}, x_{m}\right) & \preccurlyeq \sum_{j=n}^{m-1}\left[\alpha^{j} \cdot G\left(x_{0}, x_{0}, x_{1}\right)+j \alpha^{j} \cdot \varepsilon\right] \\
& \preccurlyeq \frac{\alpha^{n}}{1-\alpha} G\left(x_{0}, x_{0}, x_{1}\right)+\sum_{j=n}^{m-1} j \alpha^{j} \cdot \varepsilon \\
& \preccurlyeq \frac{\alpha^{n}}{1-\alpha} G\left(x_{0}, x_{0}, x_{1}\right)+\alpha^{n} \frac{n+\alpha}{(1-\alpha)^{2}} \cdot \varepsilon .
\end{aligned}
$$

Since $\lim _{n \rightarrow \infty} \frac{\alpha^{n}}{1-\alpha}=0$ and $\lim _{n \rightarrow \infty} \alpha^{n} \frac{n+\alpha}{(1-\alpha)^{2}}=0$ we obtain that

$$
\frac{\alpha}{1-\alpha} G\left(x_{0,}, x_{0}, x_{1}\right)+\alpha^{n} \frac{n+\alpha}{(1-\alpha)^{2}} \cdot \varepsilon \rightarrow \theta
$$

in locally convex space $E$ as $\rightarrow \infty$.

Apply Remark 2, we conclude that for every $\tau \in E$ with $\theta \ll \tau$ there exists $n_{0} \in \mathbb{N}$ such that $G\left(x_{n}, x_{n}, x_{m}\right) \ll \tau$ for all $m, n \geq n_{0}$. So $\left\{x_{n}\right\}$ is a tus-G-cone Cauchy sequence. Since $(X, G)$ is a tvs-G-cone complete metric space, $\left\{x_{n}\right\}$ is tvs-G-cone convergent in $X$ and G-cone- $\lim _{n \rightarrow \infty} x_{n}=x$. Thus, for every $\tau \in \operatorname{intP}$ and sufficiently large $n$, we have

$$
\mathcal{H}\left(T x_{n}, T x_{n}, T x\right) \preccurlyeq \alpha \cdot G\left(x_{n}, x_{n}, x\right) \cdot \alpha \frac{\tau}{3 \alpha}=\frac{\tau}{3} .
$$

Since for $n \in \mathbb{N} \cup\{0\}, x_{n+1} \in T x_{n}$, by $\left(H_{4}\right)$, we obtain that for all $n \in \mathbb{N}$ there exist $y_{n}$ $\in T_{x}$ such that

$$
G\left(x_{n+1}, x_{n+1}, y_{n+1}\right) \preccurlyeq \mathcal{H}\left(T x_{n}, T x_{n}, T x\right)+\varepsilon_{n+1} \preccurlyeq \alpha \cdot G\left(x_{n}, x_{n}, x\right)+\alpha^{n+1} \varepsilon .
$$

Then for sufficiently large $n$, we obtain that

$$
G\left(y_{n+1}, x, x\right) \preccurlyeq G\left(y_{n+1}, x_{n+1}, x_{n+1}\right)+G\left(x_{n+1}, x, x\right) \ll \frac{2 \tau}{3}+\frac{\tau}{3}=\tau,
$$

which implies G-cone- $\lim _{n \rightarrow \infty} y_{n}=x$. Since $T x$ is closed, we obtain that $x \in T x$. $\square$ Follows Theorem 1, we immediate get the following corollary.

Corollary 1 Let $(X, G)$ be a tvs-G-cone complete metric space with a solid cone $P$ and let $\mathcal{A}$ be a collection of nonempty closed subsets of $X, \mathcal{A} \neq \phi$, and let $\mathcal{H}: \mathcal{A} \times \mathcal{A} \times \mathcal{A} \rightarrow$ Ebe a tvs $-\mathcal{H}$-conemetric with respect to $G$. If the mapping $T: X \rightarrow \mathcal{A}$ satisfies the condition that exists $\alpha \in(0,1)$ such that for all $x, y, z \in X$ holds 


$$
\mathcal{H}(T x, T y, T z) \preccurlyeq \alpha \cdot G(x, y, z)
$$

\section{then $T$ has a fixed point in $X$.}

\section{Acknowledgements}

The authors would like to thank referee(s) for many useful comments and suggestions for the improvement of the article.

\section{Competing interests}

The authors declare that they have no competing interests.

\section{Received: 31 October 2011 Accepted: 26 March 2012 Published: 26 March 2012}

\section{References}

1. Huang, LG, Zhang, X: Cone metric spaces and fixed point theorems of contractive mappings. J Math Anal Appl. 322 , 1468-1476 (2007)

2. Abbas, M, Jungck, G: Common fixed point results for noncommuting mappings without continuity in cone uniform spaces. J Math Anal Appl. 341(1):416-420 (2008)

3. Jankovic', S, Kadelburg, Z, Radonevic, S, Rhoades, BE: Assad-Kirk-type fixed point theorems for a pair of nonself mappings on cone metric spaces. Fixed Point Theory Appl 2009, 16 (2009). Article ID 761086

4. Rezapour, Sh, Hamlbarani, R: Some notes on the paper "Cone metric spaces and fixed point theorems of contractive mappings". J Math Anal Appl. 345, 719-724 (2008)

5. Arshad, M, Azam, A, Vetro, P: Some common fixed point results in cone uniform spaces. Fixed Point Theory Appl 2009 11 (2009). Article ID 493965

6. Azam, A, Arshad, M: Common fixed points of generalized contractive maps in cone uniform spaces. Bull Iranian Math Soc. 35(2):255-264 (2009)

7. Bari, CD, Vetro, P: $\otimes$-pairs and common fixed points in cone metric spaces. Rend Circ Mat Palermo. 57(2):279-285 (2002)

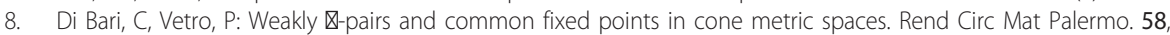
125-132 (2009)

9. Harjani, J, Sadarangani, K: Generalized contractions in partially ordered metric spaces and applications to ordinary differential equations. Nonlinear Anal. 72, 1188-1197 (2010)

10. Haghi, RH, Rezapour, Sh: Fixed points of multifunctions on regular cone metric spaces. Expo Math. 28, 71-77 (2010)

11. Kilm, D, Wardowski, D: Dynamic processes and fixed points of set-valued nonlinear contractions in cone metric spaces. Nonlinear Anal. 71, 5170-5175 (2009)

12. Kadelburg, Z, Radenovic', S, Rakočevic', V: Remarks on "quasi-contraction on a cone metric space". Appl Math Lett. 22, 1674-1679 (2009)

13. Kadelburg, Z, Pavlovic', M, Radenovic', S: Common fixed point theorems for ordered contractions and quasi-contractions in ordered cone metric spaces. Comput Math Appl. (2010)

14. Rezapour, Sh, Khandani, H, Vaezpour, SM: Efficacy of cones on topological vector spaces and application to common fixed points of multifunctions. Rend Circ Mat Palermo. 59, 185-197 (2010)

15. Rezapour, Sh, Haghi, RH: Fixed point of multifunctions on cone metric spaces. Numer Funct Anal and Opt. 30(78):825-832 (2009)

16. Du, WS: A note on cone metric fixed point theory and its equivalence. Nonlinear Anal Theory Methods Appl. 72 2259-2261 (2010)

17. Azam, A, Beg, I, Arshad, M: Fixed point in topological vector space-valued cone metric spaces. Fixed Point Theory and Appl 2010, 9 (2010). Article ID 604084

18. Beg, I, Azam, A, Arshad, M: Common fixed points for maps on topological vector space valued cone metric spaces. Int J Math Math Sci 2009, 8 (2009). Article ID 560264

19. Kadelburg, Z, Radenovic', S, Rakočevic', V: Topological vector space valued cone metric spaces and fixed point theorems. Fixed Point Theory and Applications 17 (2010). Article ID 170253

20. Kadelburg, Z, Radenovic', S, Rakočevic', V: A note on equivalence of some metric and cone metric fixed point results. Appl Math Lett. 24, 370-374 (2011)

21. Radonevic', S, Simic', S, Cakic', N, Golubovic', Z: A note on tus-cone metric fixed point theory. Math Comput Model. 54 , 2418-2422 (2011)

22. Dhage, BC: Generalized metric space and mapping with fixed point. Bull Calcutta Math Soc. 84, $329-336$ (1992)

23. Mustafa, Z, Sims, B: A new approach to generalized metric spaces. J Nonlinear Convexs Anal. 7(2):289-297 (2006)

24. Meir, A, Keeler, E: A theorem on contraction mappings. J Math Anal Appl. 28, 326-329 (1969)

25. Nadler, SB Jr: Multi-valued contraction mappings. Pacific J Math. 30, 475-488 (1969)

26. Mizoguchi, N, Takahashi, W: Fixed point theorems for multivalued mappingson complete metric spaces. J Math Anal Appl. 141, 177-188 (1989)

27. Reich, S: Fixed points of contractive functions. Boll Un Mat Ital. 5, 26-42 (1972)

28. Reich, S: Approximate selections, best approximation, fixed points, and invariant sets. J Math Anal Appl. 62, 104-113 (1978)

29. Wardowski, D: On set-valued contractions of Nadler type in cone metric spaces. Appl Math Lett. 24, $275-278$ (2011)

doi:10.1186/1687-1812-2012-52

Cite this article as: Chen: On set-valued contractions of Nadler type in tus-G-cone metric spaces. Fixed Point Theory and Applications 2012 2012:52. 